Supporting Information

\title{
Theranostic Activity of Nitric Oxide-Releasing Carbon Quantum Dots
}

Haibao Jin, Evan S. Feura, * and Mark H. Schoenfisch ${ }^{\sharp, \beta, *}$

${ }^{\dagger}$ Shanghai Key Laboratory of Advanced Polymeric Materials, School of Materials

Science and Engineering, East China University of Science and Technology,

Shanghai 200237, China

Department of Chemistry, University of North Carolina at Chapel Hill, Chapel Hill, North Carolina 27599, United States

$\S$ Division of Pharmacoengineering and Molecular Pharmaceutics, UNC Eshelman

School of Pharmacy, Chapel Hill, NC 27599, United States

*Corresponding author. E-mail: schoenfisch@unc.edu 


\section{Table of Contents:}

1. Characterization of CQDs ................................................................................

2. Characterization of NO-releasing CQDs........................................................

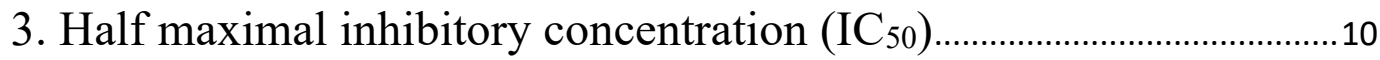

4. Elemental analysis for amine-modified CQDs.........................................10

5. In vitro cell viability of NO-releasing CQDs .............................................11

6. Fluorescence microscopy study …………………………………….............12 


\section{Characterization of CQDs}
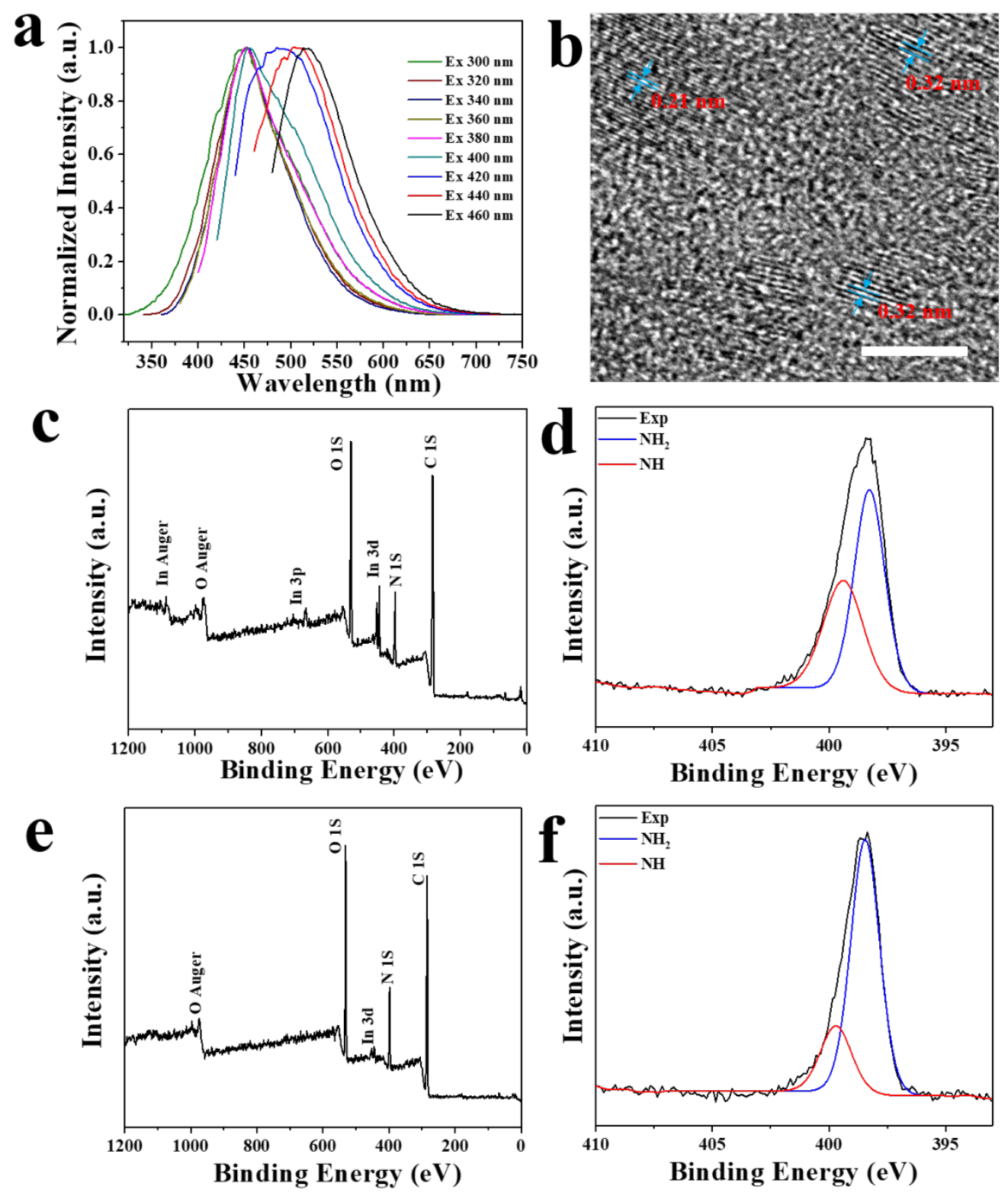

Figure S1. (a) The normalized fluorescence spectra of CQD-DA7 $(0.5 \mathrm{mg} / \mathrm{mL})$. (b) Representative high-resolution TEM images of CQD-DA7. Scale bar represents $5 \mathrm{~nm}$. (c) Full-scan XPS spectrum of CQD-DA7. (d) Curve-fitted XPS spectrum (black) of the N 1s peaks for CQD-DA7. The presence of primary (blue) and secondary (red) amines are indicated by fitted curves at binding energies of 398.2 and $399.3 \mathrm{eV}$, respectively. (e) Full-scan XPS spectrum of raw material of CD-DETA7. (f) Curve-fitted XPS spectrum (black) of the N 1s peaks for CD-DETA7. The presence of primary (blue) and secondary (red) amines are indicated by fitted curves at binding energies of 398.4 and $399.5 \mathrm{eV}$, respectively. 
Table S1. Dynamic light scattering (DLS) analysis of the CQDs.

\begin{tabular}{llcc}
\hline Materials & CQD-DA7 & CQD-HA7 & CD-DA \\
\hline Diameter $(\mathrm{nm})$ & $6.2 \pm 2.4$ & $6.4 \pm 4.4$ & $4.8 \pm 2.2$ \\
\hline
\end{tabular}

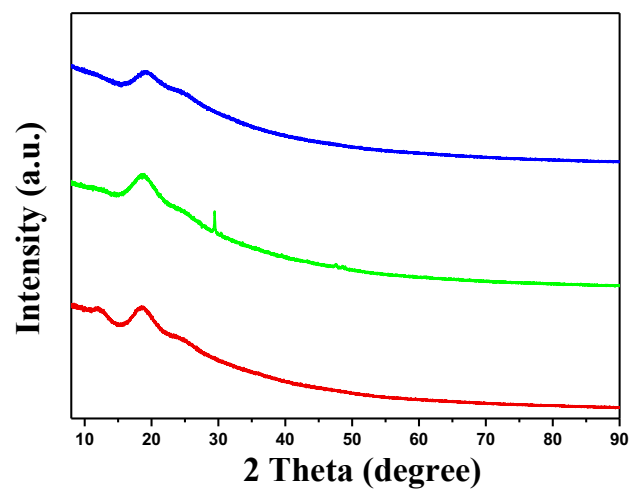

Figure S2. XRD spectra of the CQDs. Red, blue, and green lines represent the XRD spectrum of CQD-DA, CQD-HA7, and CQD-DA7. 

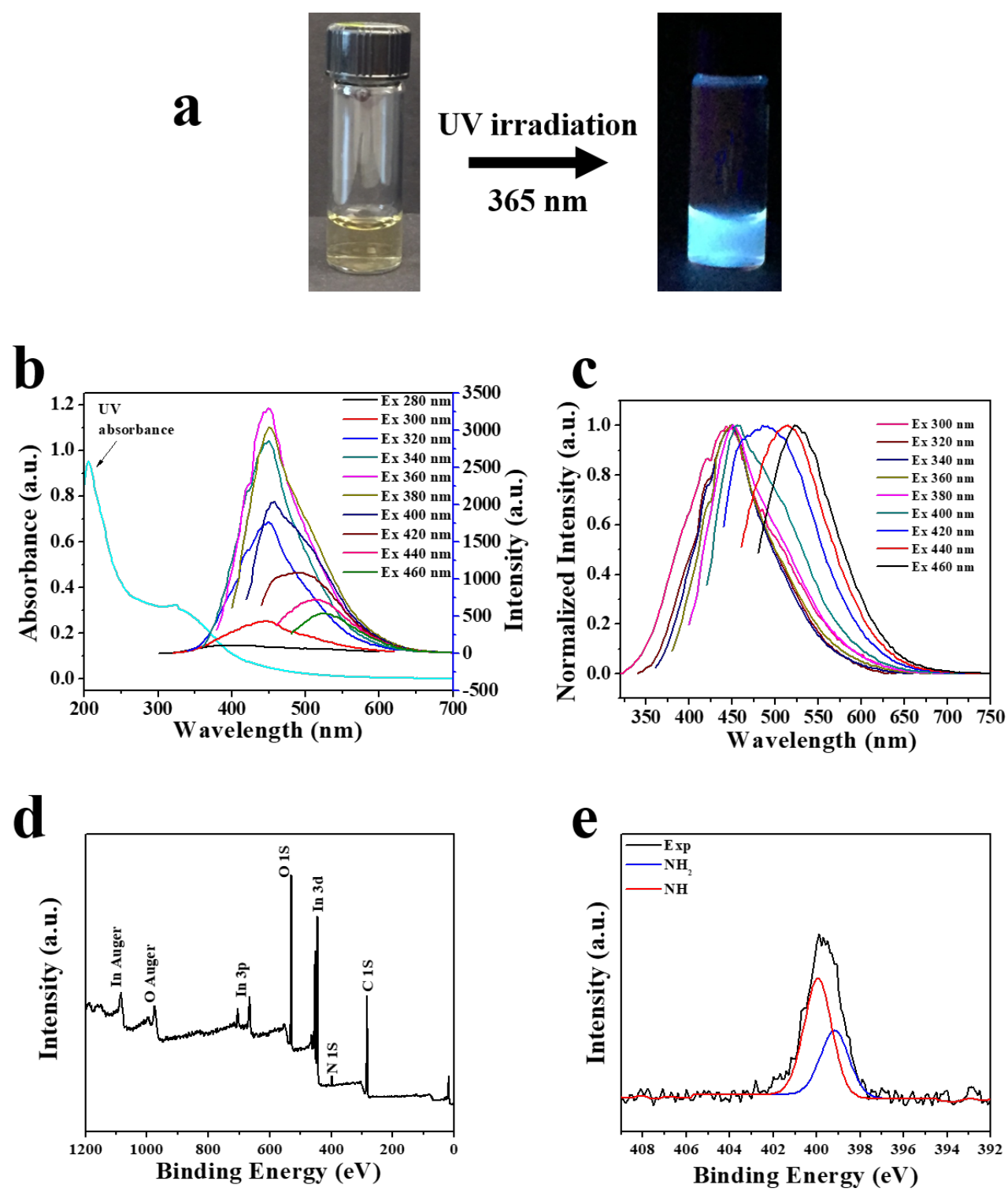

Figure S3. (a) Digital image of $1 \mathrm{mg} / \mathrm{mL}$ CQD-DA aqueous solution irradiated by UV light (365 $\mathrm{nm})$. (b) UV-Vis and fluorescence spectra of CQD-DA $(0.5 \mathrm{mg} / \mathrm{mL})$. Excitation by $360 \mathrm{~nm}$ resulted in the highest intensity emission. (c) The normalized fluorescence spectra of CQD-DA. (d) Full scan XPS spectrum of CQD-DA. (e) Curve-fitted XPS spectrum (black) of the N 1s peaks for CQD-DA. The presence of primary (blue) and secondary (red) amines are indicated by fitted curves at binding energies of 399.1 and $400.0 \mathrm{eV}$, respectively. 

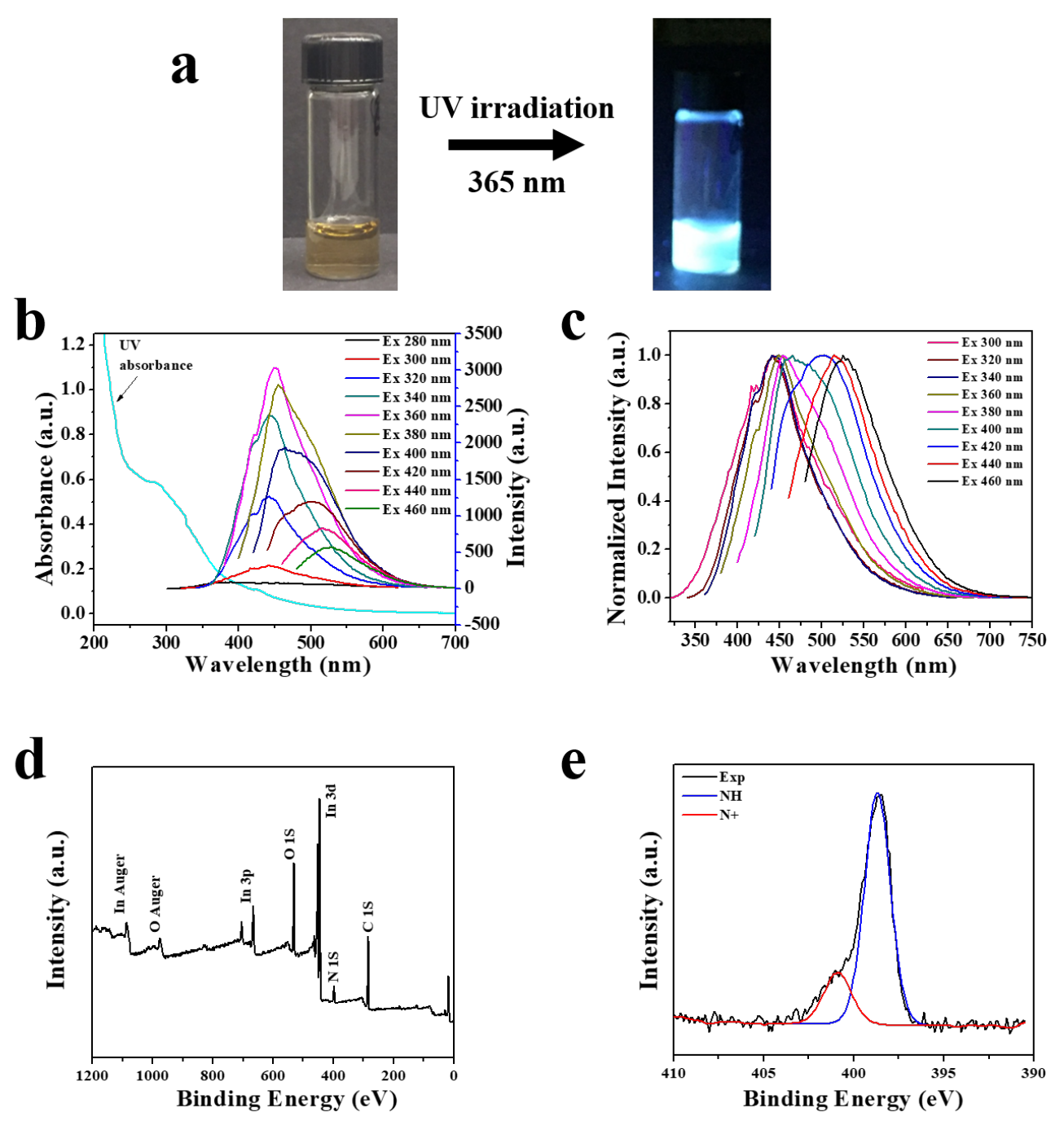

Figure S4. (a) Digital image of $1 \mathrm{mg} / \mathrm{mL}$ CQD-HA7 aqueous solution irradiated by UV light (365 $\mathrm{nm})$. (b) UV-Vis and fluorescence spectra of CQD-HA7 $(0.5 \mathrm{mg} / \mathrm{mL})$. Excitation by $360 \mathrm{~nm}$ resulted in the highest intensity emission. (c) The normalized fluorescence spectra of CQD-HA7. (d) Full scan XPS spectrum of CQD-HA7. (e) Curve-fitted XPS spectrum (black) of the N 1s peaks for CQD-HA7. The presence of secondary (blue) and charged (red) amines are indicated by fitted curves at binding energies of 398.6 and $400.9 \mathrm{eV}$, respectively. 


\section{Characterization of NO-releasing CQDs}
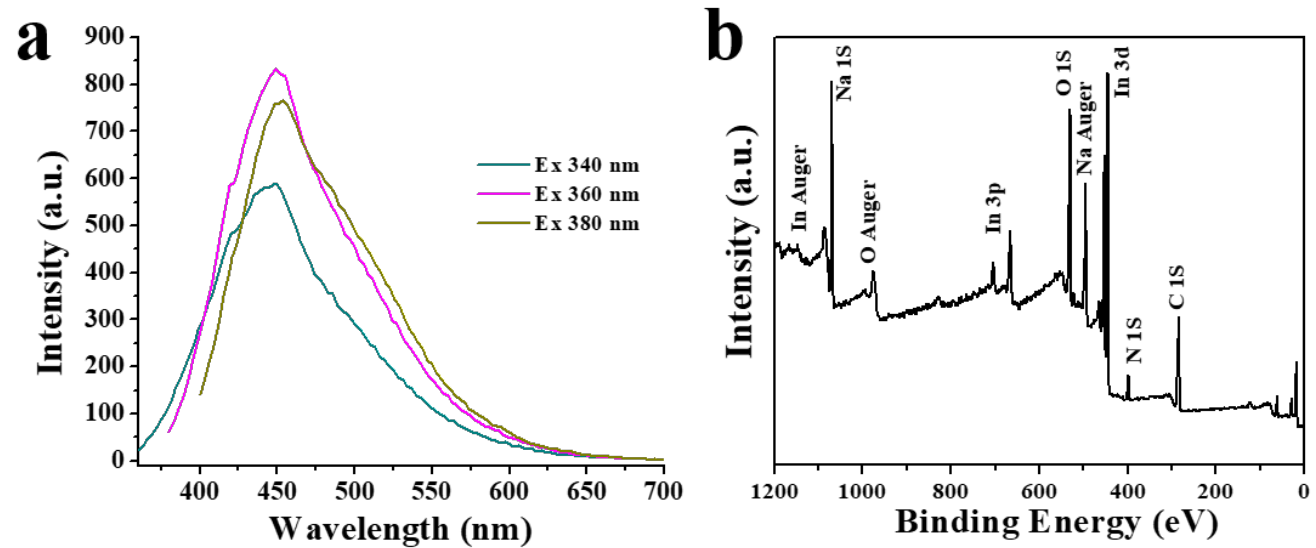

Figure S5. (a) Fluorescence spectra from CQD-DA7/NO. (b) Full-scan XPS spectrum of CQDDA7/NO. 
$\mathbf{a}$
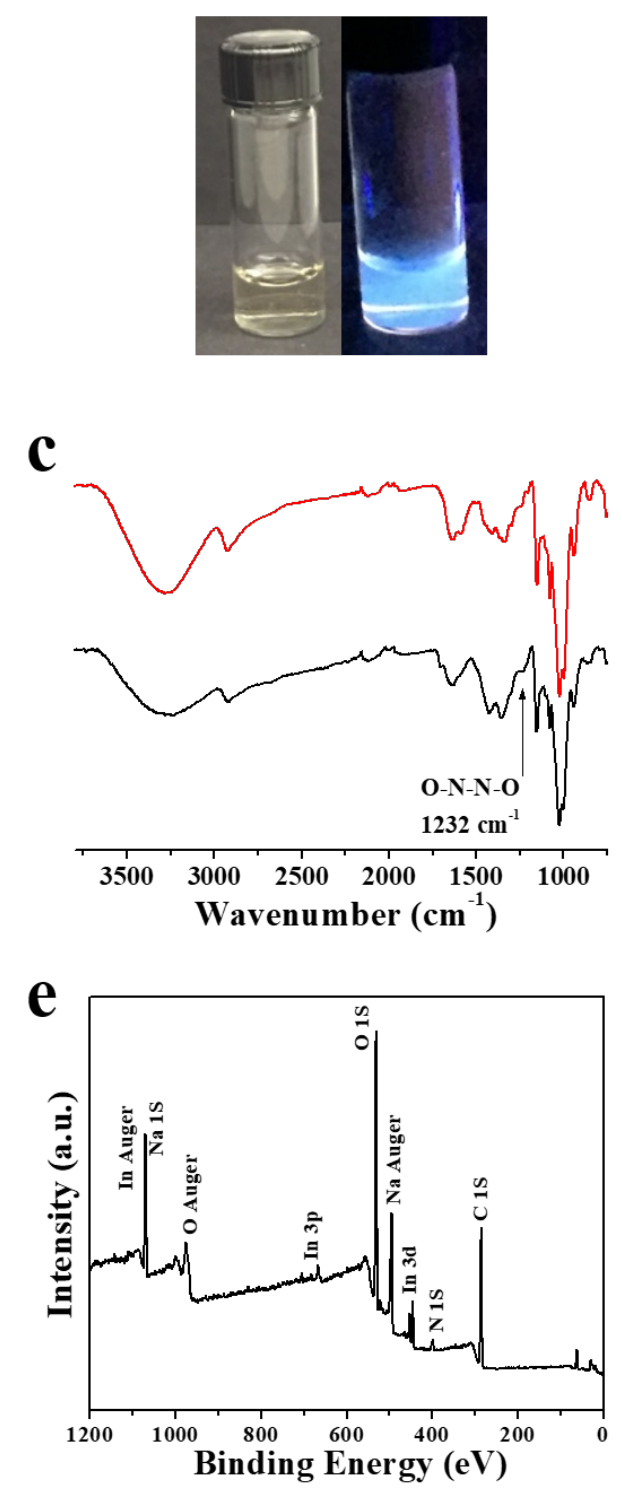
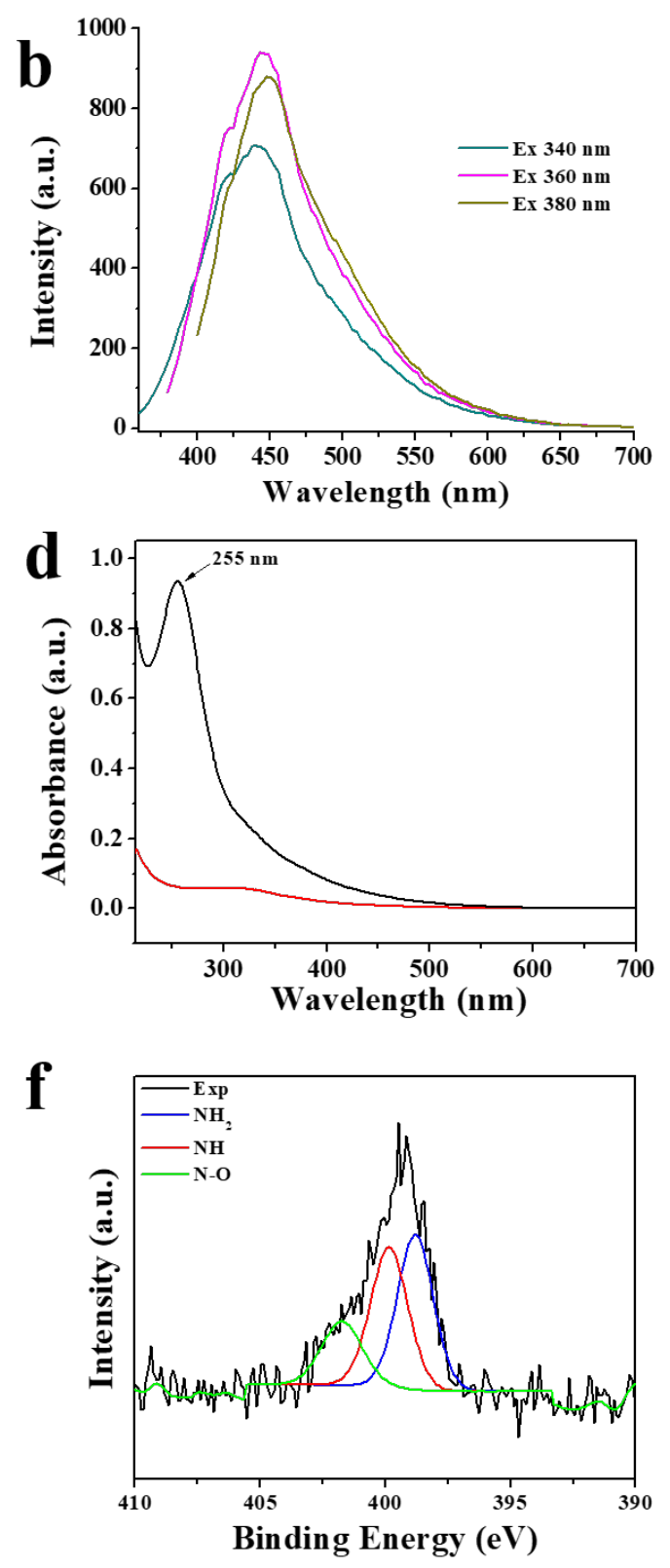

Figure S6. (a) Digital images of CQD-DA/NO aqueous solution irradiated by visible (left) and UV light (365 nm, right). (b) Fluorescence spectra of CQD-DA/NO as a function of the excitation wavelength. (c) FTIR spectra of CQD-DA (red) and CQD-DA/NO (black). (d) UV-Vis spectra of CQD-DA (red, $0.2 \mathrm{mg} / \mathrm{mL}$ ) and CQD-DA/NO (black, $0.2 \mathrm{mg} / \mathrm{mL}$ ), measured in $0.1 \mathrm{M} \mathrm{NaOH}$ at a concentration of $0.25 \mathrm{mg} / \mathrm{mL}$. (e) Full scan XPS spectrum of CQD-DA/NO. (f) Curve-fitted XPS spectrum (black) of the $\mathrm{N}$ 1s peaks for CQD-DA/NO. The presence of primary amines (blue), secondary amines (red) and N-O bonds (green) are indicated by fitted curves at binding energies of 398.7, 399.5, and $401.7 \mathrm{eV}$, respectively. 
a

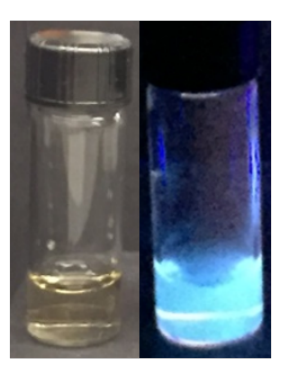

c
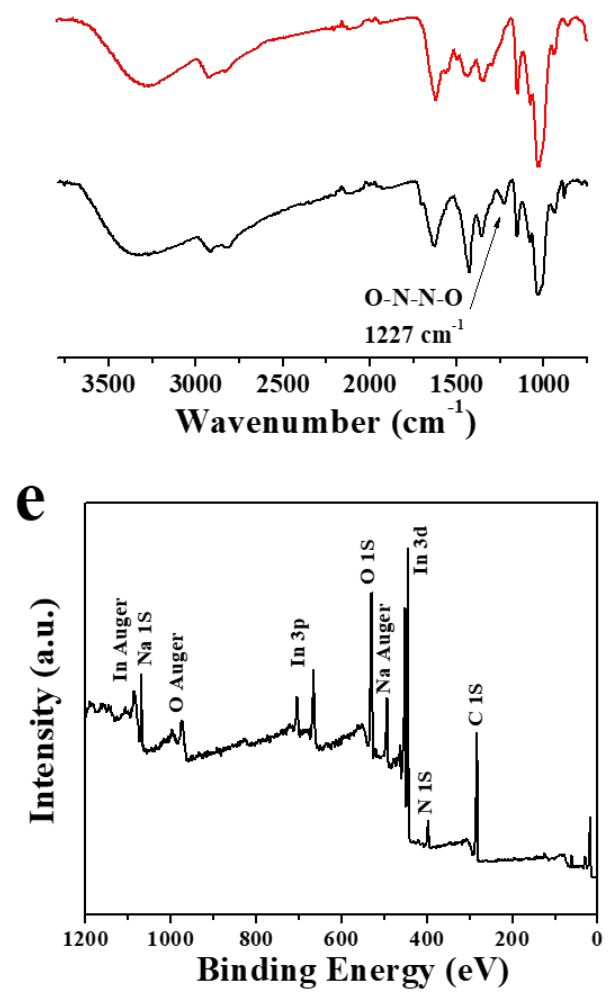
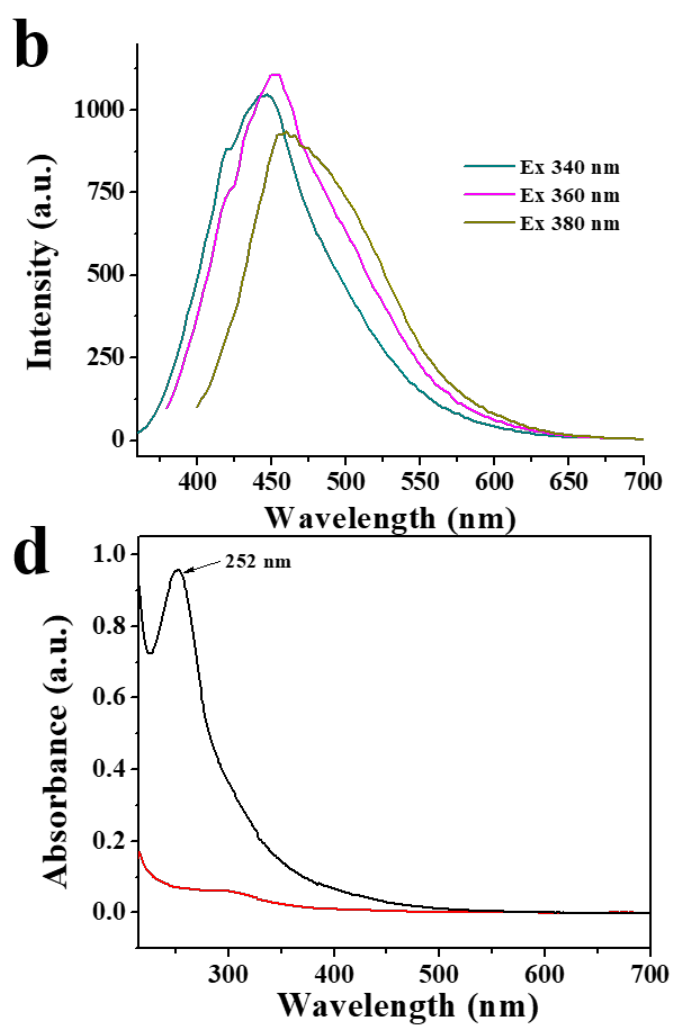

f

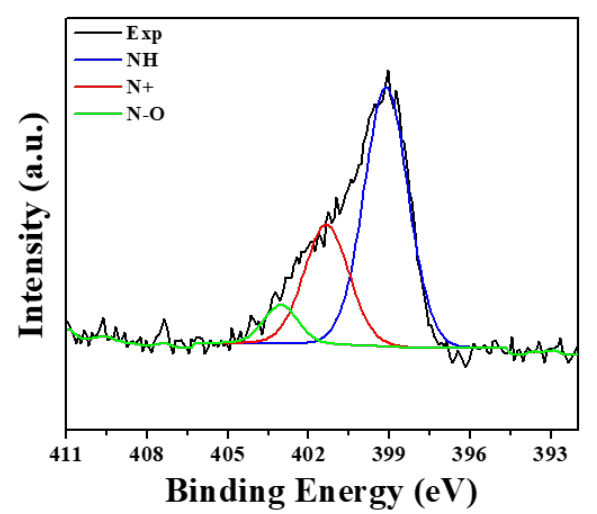

Figure S7. (a) Digital images of CQD-HA7/NO aqueous solution irradiated by visible (left) and UV light (365 nm, right). (b) Fluorescence spectra of CQD-HA7/NO as a function of the excitation wavelength. (c) FTIR spectra of CQD-HA7 (red) and CQD-HA7/NO (black). (d) UV-Vis spectra of CQD-HA7 (red, $0.125 \mathrm{mg} / \mathrm{mL}$ ) and CQD-HA7/NO (black, $0.125 \mathrm{mg} / \mathrm{mL}$ ), measured in $0.1 \mathrm{M}$ $\mathrm{NaOH}$ at a concentration of $0.05 \mathrm{mg} / \mathrm{mL}$. (e) Full scan XPS spectrum of CQD-HA7/NO. (f) Curvefitted XPS spectrum (black) of the N 1s peaks for CQD-HA7/NO. The presence of secondary amines (blue), charged amines (red) and N-O bonds (green) are indicated by fitted curves at binding energies of 399.1, 401.0, and $402.7 \mathrm{eV}$, respectively. 

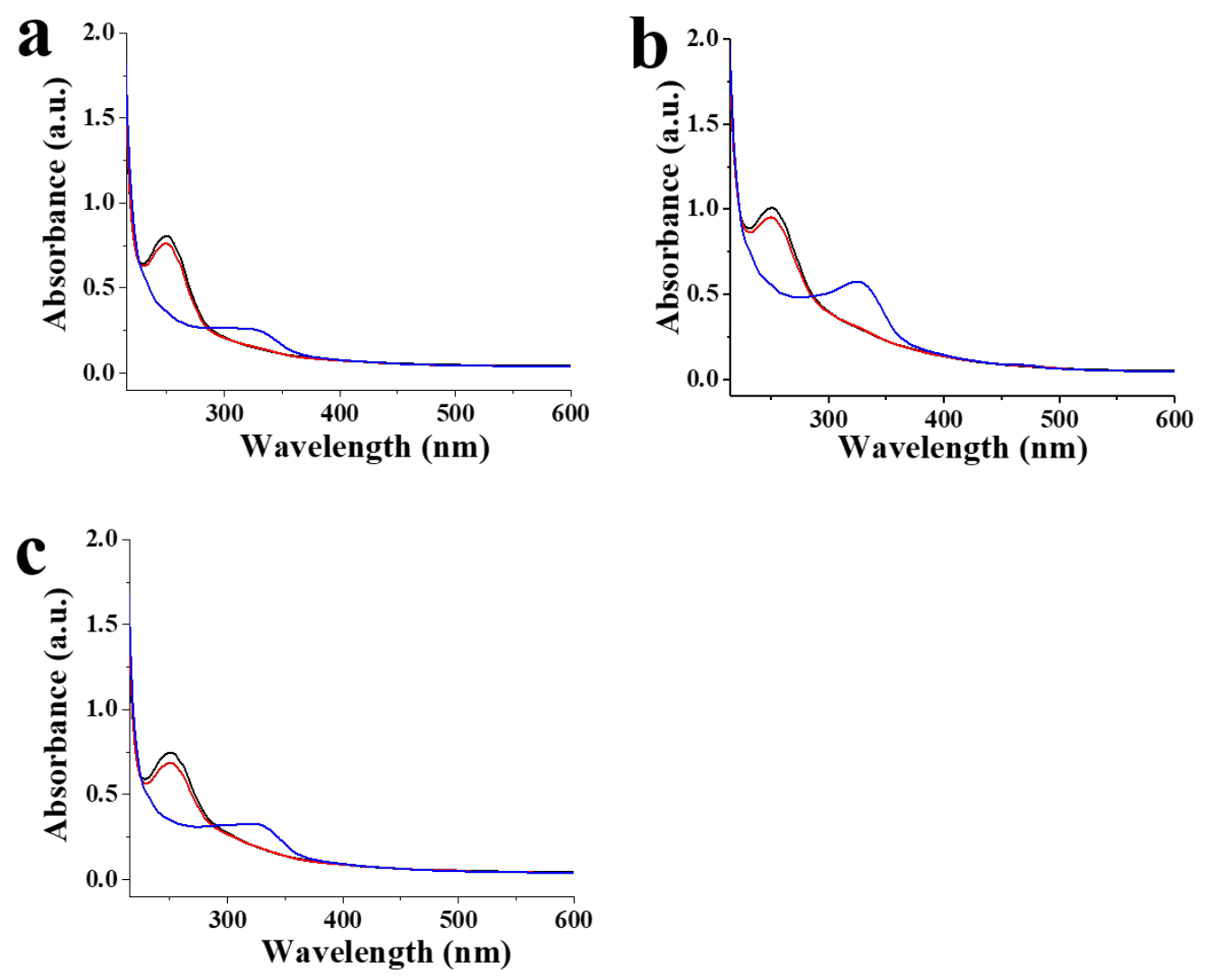

Figure S8. Stability of NO-releasing CQDs upon UV irradiation. (a) CQD-DA7/NO. (b) CQDDA/NO. (c) CQD-HA7/NO. Black line represents the UV-Vis absorption spectrum before UV irradiation. Red line represents the UV-Vis absorption spectrum after exposure to UV light (365 nm) for $15 \mathrm{~min}$. Blue line represents the UV-Vis absorption spectrum after exposure to UV light (254 $\mathrm{nm}$ ) for $15 \mathrm{~min}$. A slight decrease in the $N$-diazeniumdiolate peak was observed upon exposure of the solution to UV light $365 \mathrm{~nm}$, likely due to initiation of NO release in aqueous solution. UV 254 $\mathrm{nm}$ exposure resulted in significantly greater NO release and loss of the $\mathrm{N}$-diazeniumdiolate UVVis absorption feature. 
3. Half maximal inhibitory concentration $\left(\mathrm{IC}_{50}\right)$

Table S2. $\mathrm{IC}_{50}$ of NO-releasing CQDs toward different cell lines

\begin{tabular}{ccccc}
\hline \multirow{2}{*}{ Materials } & \multicolumn{4}{c}{$\mathrm{IC}_{50}, \mathrm{mg} / \mathrm{mL}$} \\
\cline { 2 - 5 } & Pa14C & A549 & SW480 & HPNE \\
\hline CQD-HA7 & 2.93 & 1.48 & 1.78 & 6.94 \\
CQD-HA7/NO & 1.37 & 0.86 & 0.72 & 2.67 \\
CQD-DA7 & 2.27 & 1.53 & 1.10 & 5.68 \\
CQD-DA7/NO & 0.78 & 0.25 & 0.47 & 1.29 \\
CQD-DA & 5.53 & 3.16 & 3.16 & 8.92 \\
CQD-DA/NO & 6.38 & 2.76 & 2.55 & 7.20 \\
\hline
\end{tabular}

\section{Elemental analysis for amine-modified CQDs}

Table S3. Elemental analysis characterization of the secondary amine-functionalized CQDs

\begin{tabular}{cccc}
\hline Materials & $\mathrm{C}(\%)$ & $\mathrm{H}(\%)$ & $\mathrm{N}(\%)$ \\
\hline CQD-DA & 42.05 & 6.19 & 1.63 \\
CQD-DA7 & 45.67 & 8.62 & 13.16 \\
CQD-HA7 & 45.21 & 7.80 & 9.29 \\
\hline
\end{tabular}



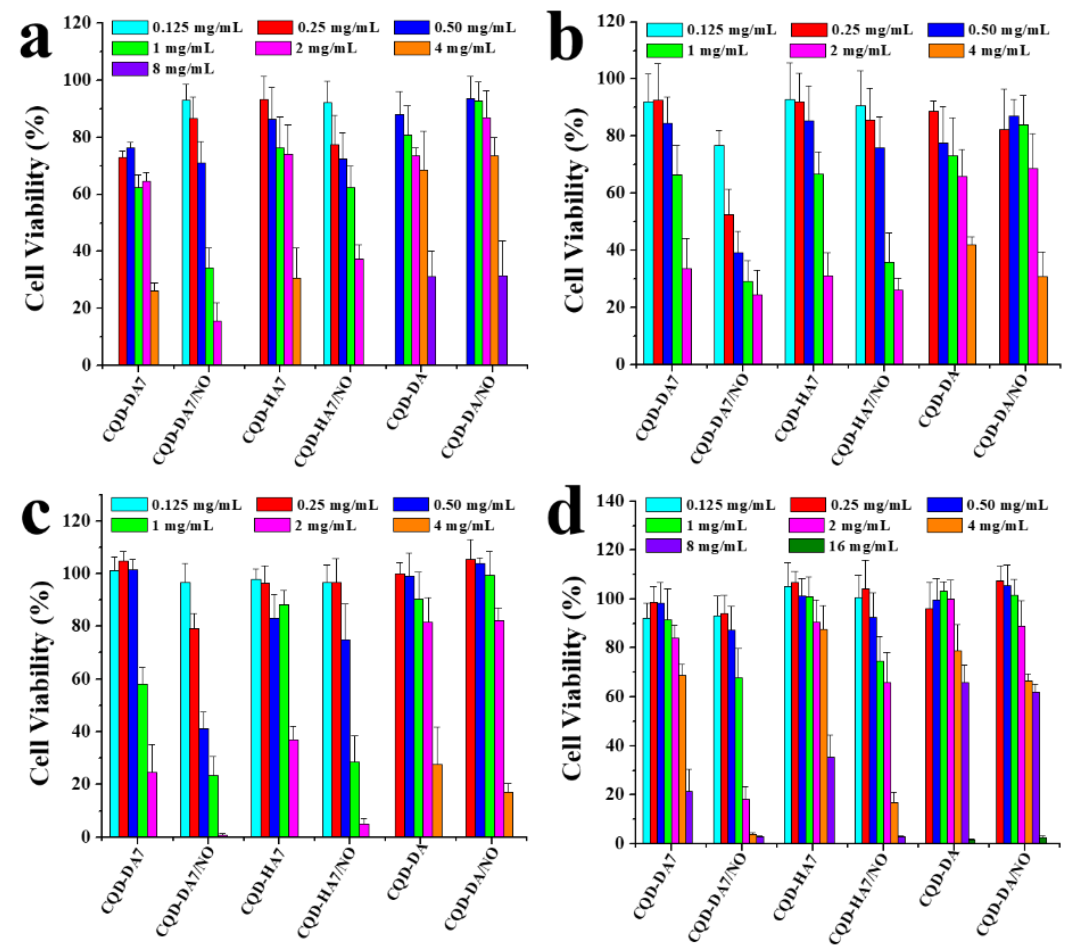

Figure S9. In vitro cell viability was evaluated using the MTS assay after treating (a) Pa14c pancreatic, (b) A549 lung, (c) SW480 colorectal cancer cells, and (d) healthy human epithelial cells (HPNE) with control (i.e., non-NO-releasing) and NO-releasing CQDs for $24 \mathrm{~h}$ as a function of the CQD concentration. The data represents the mean standard deviation of at least three determinations. 


\section{Fluorescence microscopy study}

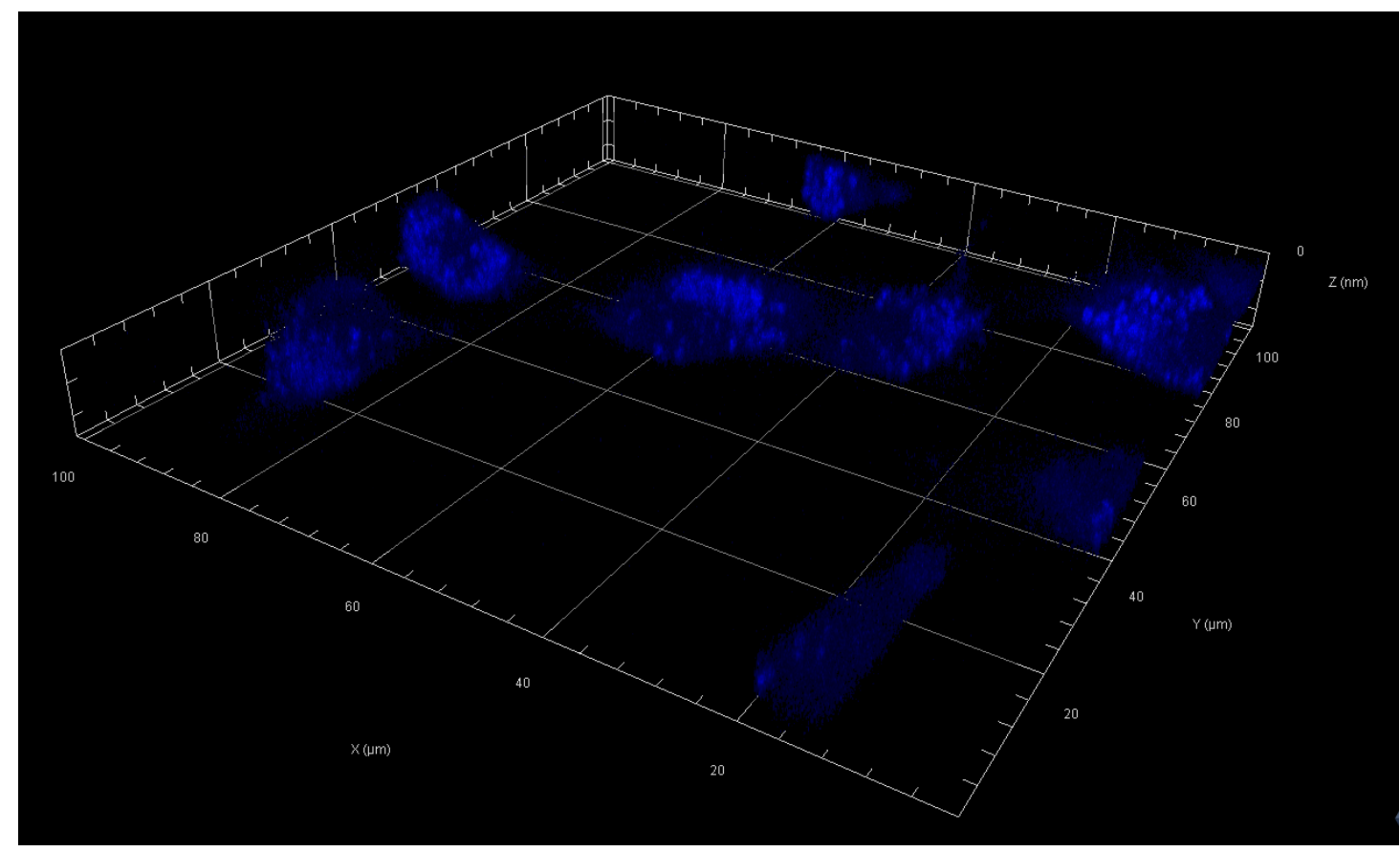

Figure S10. 3D fluorescence imaging of A549 cells after treatment with CQD-DA7/NO (405 nm excitation wavelength) evaluating the primary distribution of CQD-DA7/NO in the cell membrane and cytoplasmic areas.

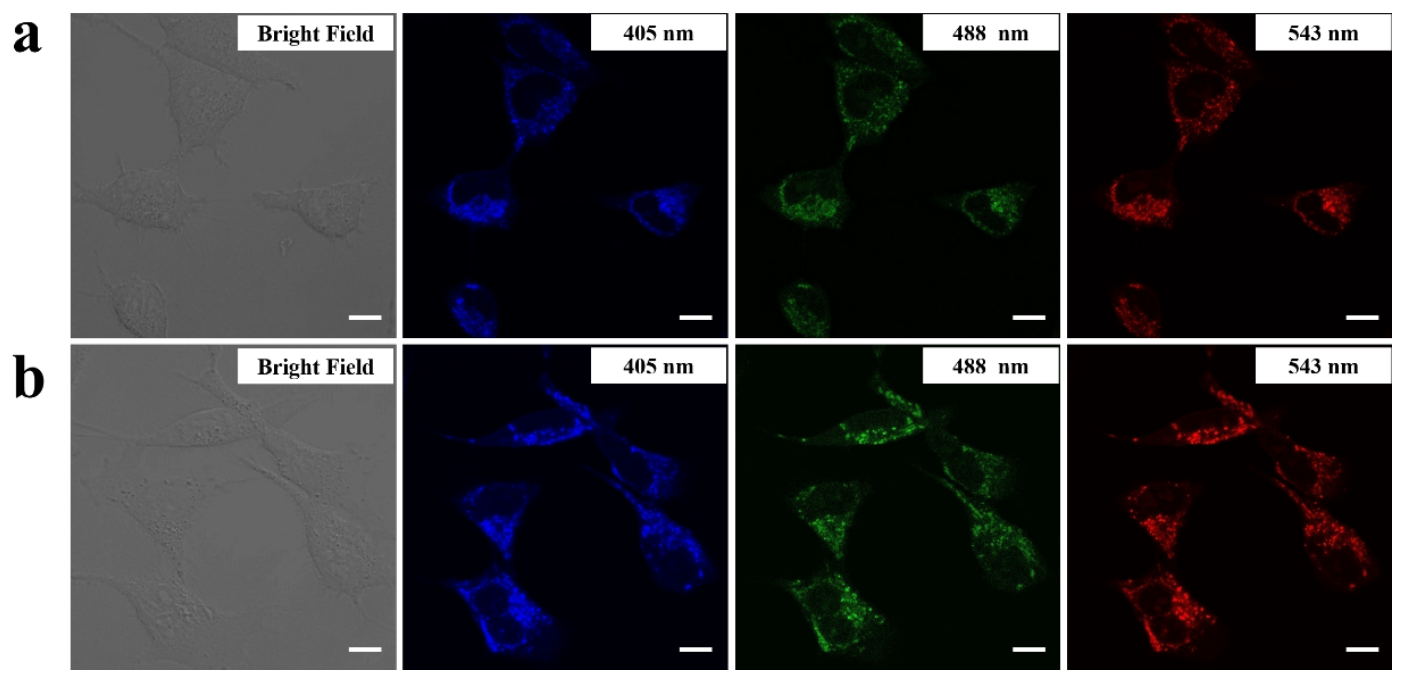

Figure S11. Optical (bright field) and confocal laser scanning microscopy images of A549 cancer cells treated with CQD-DA/NO (a) and with CQD-HA7/NO (b) for $24 \mathrm{~h}$ using 405, 488, and 543 $\mathrm{nm}$ excitation wavelengths. Scale bars in each image represent $10 \mu \mathrm{m}$. 